\title{
Temporal and spatial features in detecting one- and two-dimensional constraints in complementary visual displays*
}

\author{
IRWIN POLLACK \\ Mental Health Research Institute, University of Michigan, Ann Arbor, Michigan 48104
}

\begin{abstract}
Spatial complements of visual displays of one- and two-dimensional Markov spatial constraints were successively presented for discrimination. Although each complement alone was sufficient for discrimination, spatial complements interfere when presented successively in time. Substantial changes in performance are observed for temporal separations as short as $10 \mathrm{msec}$ and for spatial separations as short as $1 \mathrm{~min}$ in visual angle. Complete processing appears to take place in the interval between successive displays: thresholds are constant for a constant sum of display duration plus the interfield interval. Implications of complementary interference for a general theory of visual masking are briefly discussed.
\end{abstract}

Three paradigms characterize classes of experiments dealing with the interaction of successively presented display fields: the masking paradigm, where an information field is presented before, after, or between noninformational or "noise" fields; the Eriksen-Collins (1967) paradigm, where the elements of an informational field are subdivided between two display fields; and the Mayzner-Greenberg paradigm (1971), in which successively presented fields are spatial complements of each other. Performance suffers in the masking paradigm as the interfield interval, IFI, is made shorter and shorter. The interaction of the noninformational materials with the informational materials has been described by a variety of processes, ranging from luminance summation by contrast levels (Eriksen, 1966) to the interruption of the information processing devoted to the display materials (Sperling, 1963). By contrast, performance improves in the Eriksen-Collins paradigm as IFI is shortened, as related elements are presented in spatial-temporal proximity. And, as in the masking paradigm, performance suffers in the Mayzner.Greenberg complementary paradigm at short interfield intervals, although each complement field alone is sufficient for task solution. Interaction processes of the masking paradigm which depend upon spatial overlap between fields (Uttal, 1970) are not directly relevant to the complementary paradigm because of the lack of spatial overlap.

In almost all of the previous studies, the informational fields consisted of alphabetic characters. Such symbols have the striking advantage that training time is reduced. However, the number of relevant display elements studied is often small. and inferences with respect to the spatial microstructure of such displays are often difficult to make.

*The research was supported in part by Grant GB X14036X from the National Science Foundation. The author wishes to acknowledge the assistance of Nancy Mandell for supervision of the experimental tests. Louis Wojnaroski for the PDP.9 computer program. and Robert Shea for processing the experimental results.
The present tests attempt to extend the complementary-field paradigm to displays that are directly defined in terms of their spatial microstructure, and to displays in which performance can also be defined in terms of stimulus measures necessary to achieve a fixed level of performance. In addition, such displays can be generated by computational algorithms and may provide a richer test bed to examine spatial-temporal characteristics than do alphabetic displays.

\section{METHOD}

\section{Displays}

A single display cycle consisted of the following events. Four matrices. arranged in a 2 by 2 format, were painted upon an $8 \times 8 \mathrm{~cm}$ display surface (Tektronix 602 equipped with a fast P-15 phosphor) by a PDP-9 computer. Three of the matrices employed a reference constraint level; one employed a different constraint level. The position of the "odd" matrix was varied at random. A display of four matrices, requiring a painting duration of $\mathrm{d} \mathrm{msec}$, was replotted $\mathrm{n}_{2}$ successive times for a display duration $D=n_{1} d$ msec. Following a wait of $W$ msec. the complement of the first display was plotted $n_{2}$ successive times. The entire display cycle was then replicated $R$ times for a total duration of $T$ sec, where $T=R\left(n_{1} d+W+n_{2} d+W\right)$. Following the $O$ 's response and feedback, an independently generated display cycle was initiated. Sequences were first calculated and then painted by a high-speed plotting routine at $9 \mu$ sec per plotted point. Binary sequences were encoded into sequences of dots and nondots and were painted upon imaginary matrices with an interdot spacing of $1.17 \mathrm{~mm}$. On the average. four 32 by 32 matrices with a dot and no-dot probability $=0.5$ were painted in $4 \times 1.024 \times 0.5 \times 9 \mu \mathrm{sec}$, or about $18 \mathrm{msec}$. Displays were painted in a fixed geographical sequence. The minimum interdot interval. IDI, between a display and its spatial complement was the painting interval for a single display, $d$.

\section{Constraints}

Displays with one-dimensional constraints were achieved by varying the conditional repetition probability $P(A \mid A)$ of the generated sequences. When translated into the display. high P $(A, A)$ levels plot long runs of dots and of nondots: low $P(A \mid A)$ levels plot atternations of dots and of nondots. Displays with two-dimonsional constraints were ahieved by varying the 


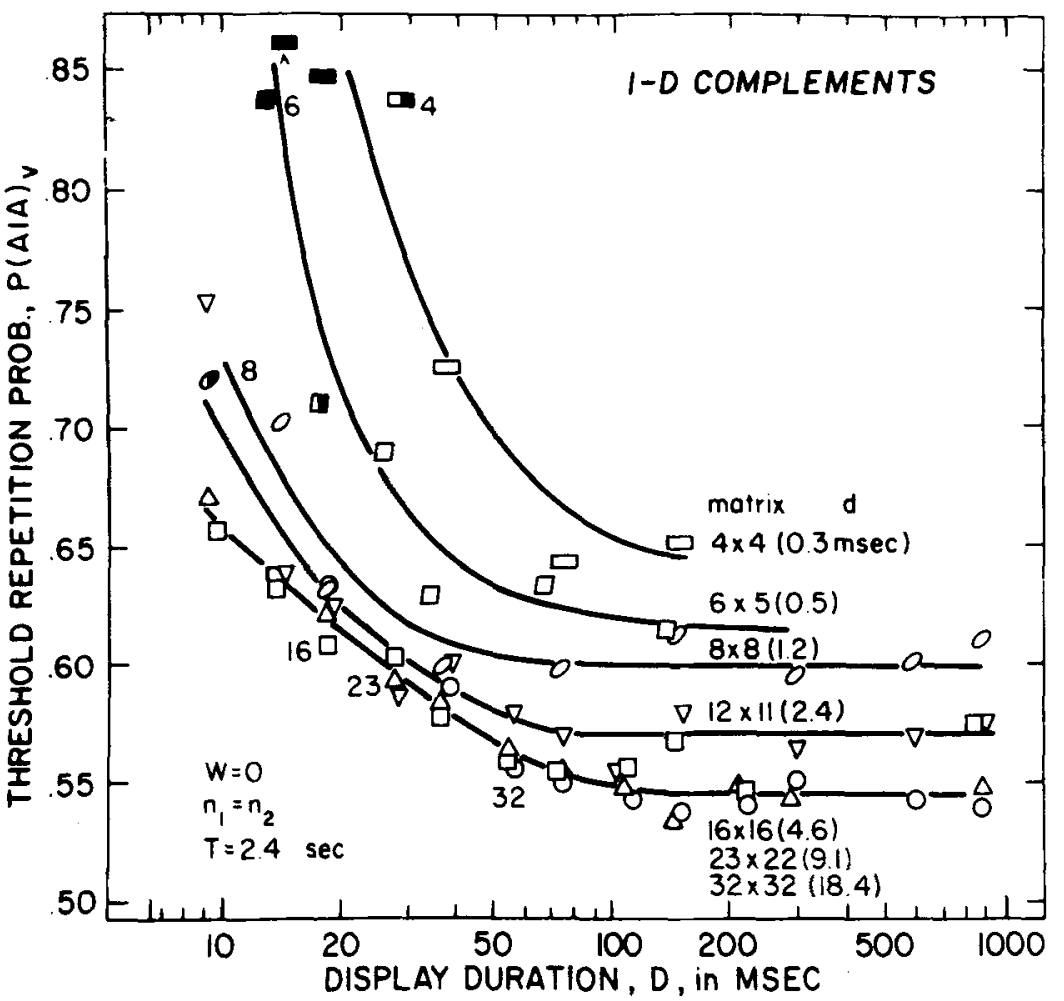

Fig. 1. One-dimensional constraint thresholds for repeatedly presented complementary pairs. The parameter is the size of the matrix and the plotting time for a single painting of each complement. The abscissa in Figs. 1, 3, and 5 is the duration of each complement, achieved by successively repainting the display. The additional waiting time between complements in Figs. 1-4 was 0 . The sequence of complementary pairs was repeatedly plotted in Figs. 14 for a constant total observational time of $2.4 \mathrm{sec}$. In Figs. 1, 2, and 9, the one-dimensional reference transitional constraint, $\mathbf{P}(\mathbf{A} \mid \mathbf{A})_{\mathbf{R}}$, equaled 0.50 . probability of an even aimed-for parity sum, $\mathrm{P}\left(\mathrm{S}_{\mathbf{e}}\right)$, within local 2 by 2 sections of the matrix. At high $P\left(S_{e}\right)$ levels, displays with blocks of dots or nondots result; at low $P\left(S_{e}\right)$ levels, displays of even texture result. (Further details are given in Pollack, 1972.)

\section{Procedure}

A single observational sequence consisted of four matrices: three at a reference constraint level, $\mathrm{P}(\mathrm{A} \mid \mathrm{A})_{\mathbf{R}}$ or $\mathbf{P}\left(\mathrm{S}_{\mathbf{e}}\right)_{\mathbf{R}}$, and one at a variable constraint level, $\mathrm{P}(\mathrm{A} \mid \mathrm{A})_{\mathrm{V}}$ or $\mathrm{P}\left(\mathrm{S}_{\mathrm{e}}\right)_{\mathrm{V}}$. O's task was to select the odd display in the four-alternative forced-choice (4AFC) test. He used a response box with four buttons arranged in the same 2 by 2 format as the display. An adaptive stimulus programming procedure varied $P(A \mid A)_{V}$ in the 1-D tests, or $P\left(S_{e}\right)_{V}$ in the 2-D tests, to converge upon $50 \%$ correct response in the 4AFC test. Under difficult viewing conditions, the adaptive procedure sought probability levels below 0 and beyond 1.0. A trial was provided under the extreme probabilities; an incorrect response terminated the trial. With $\leqslant 10 \%$ terminated trials, thresholds represent the geometric means across conditions. With $>10 \%$ terminated trials, thresholds represent median scores, including the terminated trials. With $10 \%-20 \%$ terminated trials, thresholds are plotted as half-filled points; with $>20 \%$ terminated trials, thresholds are plotted as filled points. A rough measure of difficulty is the proportion of terminated trials, as represented by the degree of shading of the points. The Os were specifically requested not to blink repeatedly or to wave their fingers before the display. These operations often served to isolate the component displays of a uniform field formed by the display and its complement.

\section{Observers}

The Os were university students, highly experienced in forced-choice testing. Natural binocular viewing was employed. $O$ could adjust his distance to the display for clarity and comfort. The median distance was about $75 \mathrm{~cm}$. Each of $16 \mathrm{Os}$ contributed two thresholds over 624 experimental conditions for a total of $0.2 \times 10^{5}$ thresholds

\section{RESULTS}

\section{Temporal Factors}

\section{One-Dimensional Constraints}

Figure 1 presents thresholds for repeated display cycles with no additional waiting time. Display duration was controlled by repeated painting of each display field. The parameter is the size of the matrix and the time in milliseconds for plotting a single field of four matrices or for its paired complement. The interdisplay interval, IDI, equalled the display duration, $D=n_{1} d=$ $\mathrm{n}_{2} \mathrm{~d}$, since there was no additional waiting time, $W$. The total display duration of $2.4 \mathrm{sec}$ was attained by successively repeating the cycle of painting complementary pairs.

As indicated by the high thresholds and by the shaded points, discrimination is difficult for extremely short interdot intervals. Discrimination deteriorates for IDIs below $20 \mathrm{msec}$ for small matrices; but extremely large threshold changes are not obtained for the larger matrices down to IDIs of $10 \mathrm{msec}$.

The rapid change in thresholds can be averted by providing brightness differences between the display and its complement. Figure 2 presents results in which the display field and its complement were repeatedly 
painted an unequal number of times. $n_{1} \neq n_{2}$. within each cycle of the complementing pairs. Assuming that display intensity is roughly proportional to the number of repeated paintings, an intensity ratio of 1.6 is required to achieve that performance characteristic of a controf condition without the complement. The apparent leveling of $f$ and further decreases in thresholds at low $n_{1} / n_{2}$ ratios is artifactual: to obtain an $n_{1} / n_{2}$ ratio of 1.1 , for example. 11 successive paintings of a display were followed by 10 successive paintings of the complement. In such cases, the duration of each display field may be sufficient-as shown in Fig. 1 with $n_{1}=n_{2}$-to attain excellent discrimination in the absence of brightness differences.

\section{Two-Dimensional Constraints}

Two-dimensional constraint thresholds are far more sensitive to the effect of complementary displays than are 1-D constraint thresholds. Figure 3 parallels the organization of Fig. 1 in presenting the results of tests with no additional wait between the display and its complement. Thresholds rise rapidly below $25 \mathrm{msec}$ for all size matrices, although small matrices remain more sensitive to the effect of display duration.

Two-dimensional constraints also require a larger differential intensity ratio for complementary display fields. Figure 4 presents the results of tests where $n_{1} \neq n_{2}$. Thresholds increase for intensity ratios below $3: 1$ to $4: 1$, and increase rapidly in the same region where 1-D constraints are barely affected.

The effect of introducing an additional waiting delay,

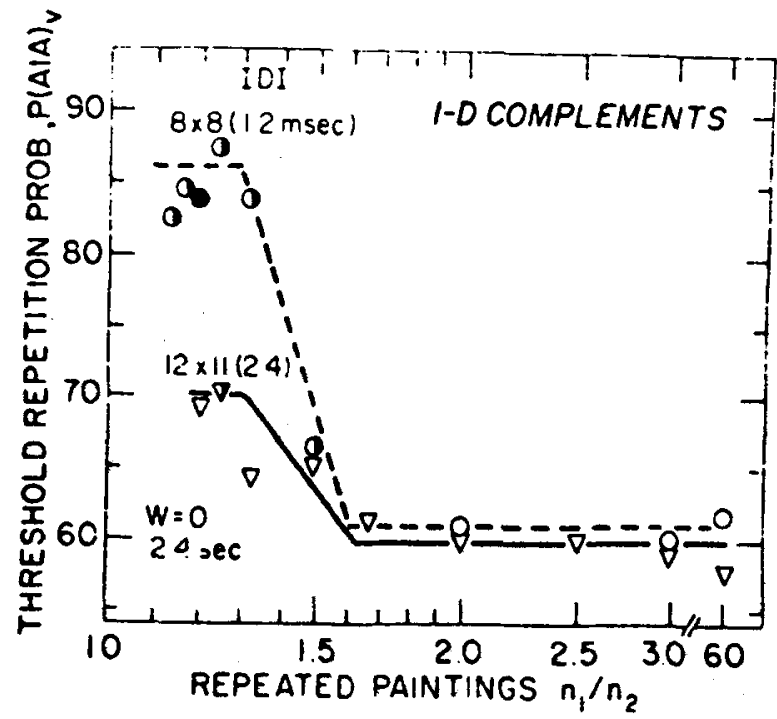

Fig. 2. One-dimensional constraint thresholds for repeatedly presented complementary pairs. The parameter is the size of the matrix and the plotting time for a single painting of each complement. The abscissa in Figs. 2 and 1 is the ratio of the number of paintings of the two display fields in which $n$, and $n$. are small integers consistent with the $n, n$, ratio permits. For example, for $n_{1} / n_{2}=2.0 . n_{1}=2$ and $n_{2}=1$ : for $n_{1} / n_{2}=1.5, n_{1}$ $=3$ and $n_{2}=2:$ for $n_{1} n_{2}=1.14, n_{1}=8$ and $n_{1}=7$.

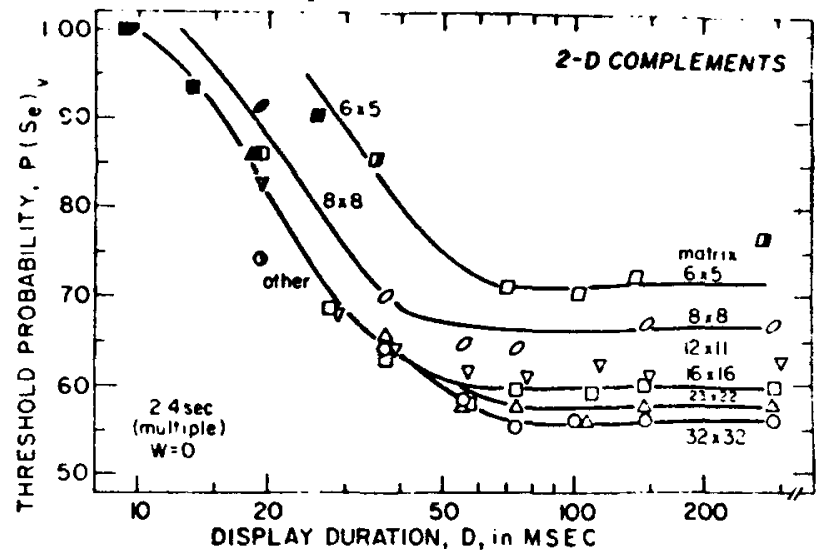

Fig. 3. Two-dimensional constraint thresholds for repeatedly presented complementary pairs. Organization is as in Fig. 1. In Figs. $3-8$ and 10 , the two-dimensional reference transitional constraint, $P\left(S_{e}\right)_{R}$, equaled 0.50 .

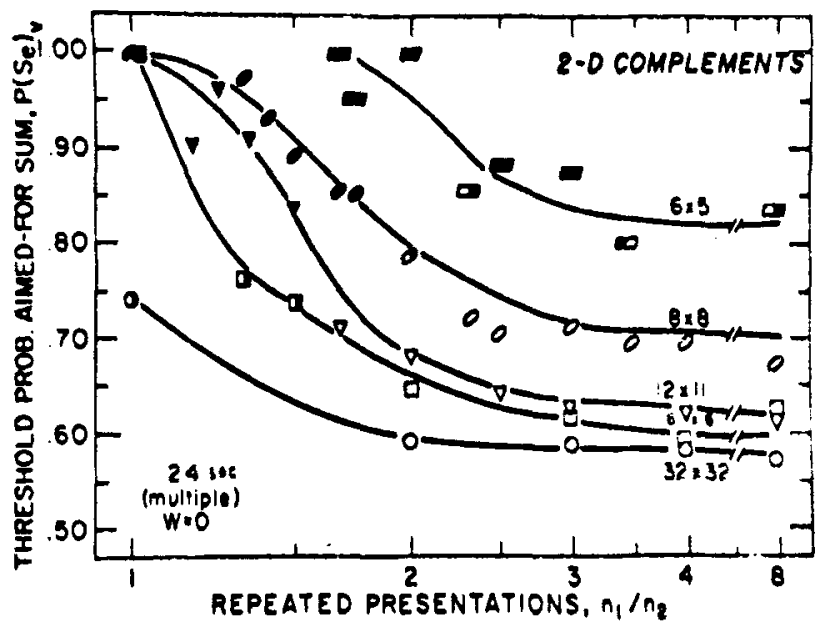

Fig. 4. Two-dimensional constraint thresholds for repeatedly presented complementary pairs. Organization is as in Fig. 2.

W, for a single presentation of each of the complementary fields is shown in Figs. 5 and 6. Figure 5 considers the proportion of terminated trials as a function of the waiting duration. The left panel ignores the duration required to generate the display; the right panel replots the data of the left panel in terms of the entire interdot interval. including the display duration and the waiting interval. In terms of the latter measure, the extreme spread of the results in the left panel becomes markedly reduced in the right panel. The thresholds of Fig. 6 lead to the same conclusion: the wide spread of thresholds as a function of the waiting interval is reduced in terms of the total interdot interval. This conclusion must be qualified by the use of the logarithmic scale which expands the left section of the left panel. As before. thresholds increase markedly below IDI $=25 \mathrm{msec}$. and smaller matrices are more sensitive to IDI.

The fine sensitivity to the waiting period between 

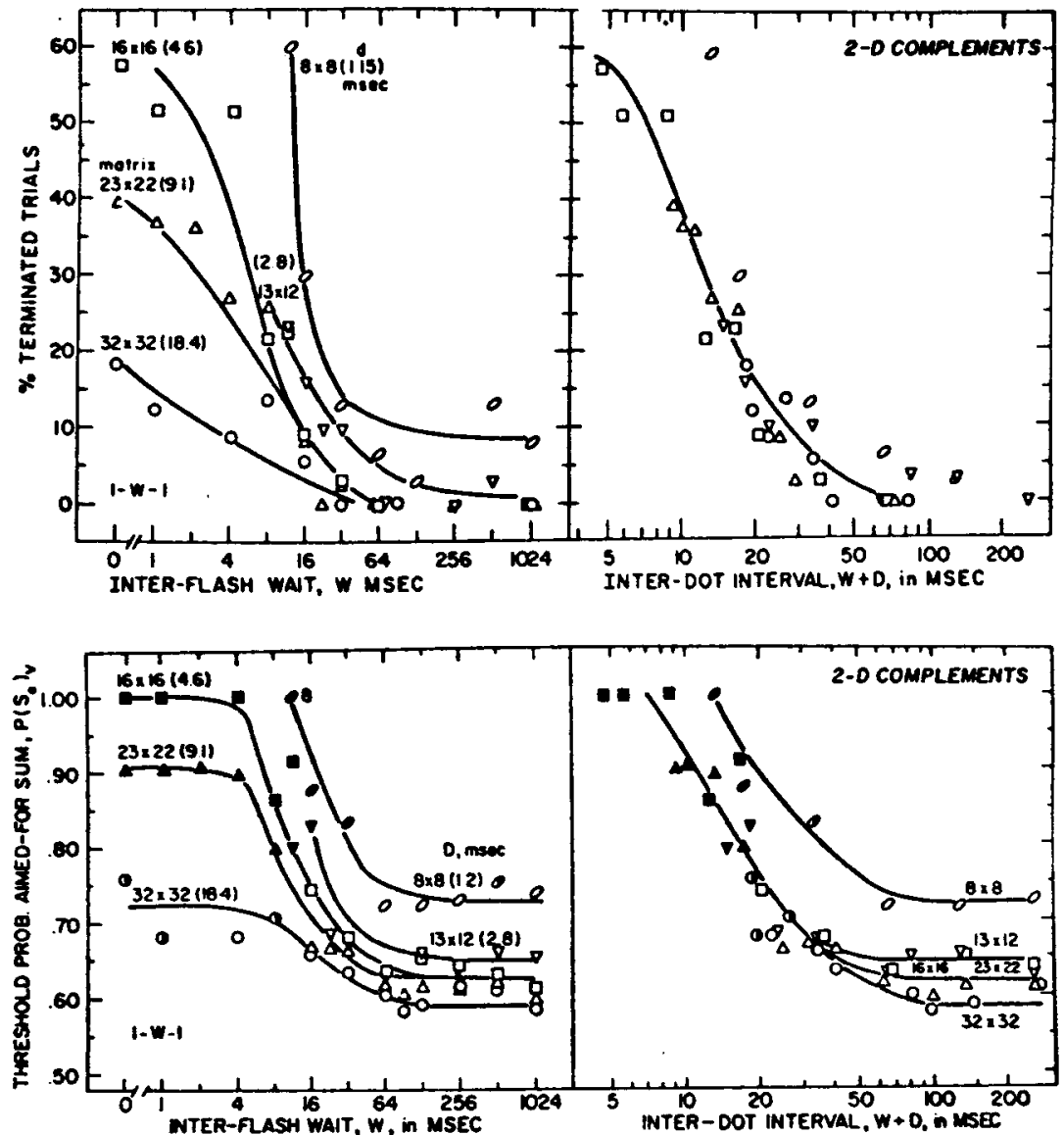

Fig. 5. Proportion of terminated trials-a crude measure of discriminability - for single presentations of two-dimensional constraints within complementary pairs. The abscissa of the left section is the additional waiting interval between complements. The data are replotted in the right section in terms of the total interdot interval, which includes the painting time of the first display plus the wait interval.
Fig. 6. Two-dimensional constraint thresholds for single presentations of complementary pairs. Organization is as in Fig. 5.

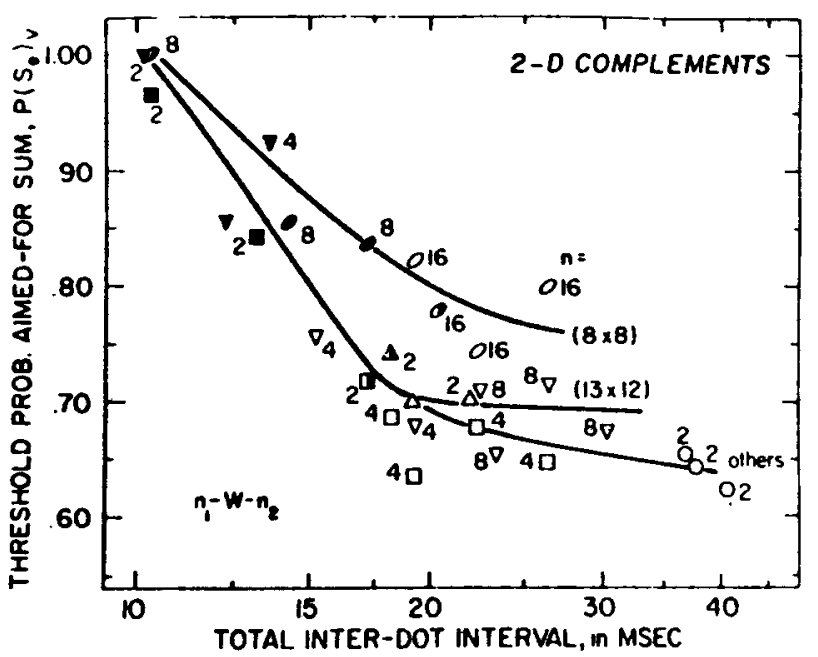

Fig. 7. Two-dimensional constraint thresholds for nonrepeated presentations of complementary pairs. The shape of the points reflects the size of the matrix, as coded in Fig. 6. The parameter is the number of successive repaintings of each complementary pair. The abscissa, as in the right section of Fig. 6 , is the sum of the display duration plus the interdisplay waiting interval.

successive complementary display fields is maintained with repeated paintings of each display field. Figure 7 considers such thresholds with the coordinates of the right panel of Fig. 6 . The shapes of the points reflect the matrix sizes, as in Figs. 3-6; the numerals associated with each point represent $n_{1}=n_{2}$, the number of successive paintings of each field.

Finally, we can compare performance for a given field and for a pair of complementary fields when paired complements are flanked by a form of interference. Figure 8 represents two sets of tests. In the $x-n_{1}-x$ tests, a 32 by 32 display field was plotted $n_{1}$ successive times and was either preceded and/or followed by the masking sequence of one painting of the display field immediately followed by its complement. The $x$ refers to a binary code where: 1 = masking sequence before the display field, 2 = masking sequence after the display field, 3 = masking sequence both before and after the display field, and $0=$ no additional displays. For example, in the $x-n_{1}-x$ tests, with $x=3$ and $n_{1}=2$, the sequence of Display Field $A$ and its complement, $A^{\prime}$, was $A^{\prime} A^{\prime} A$ A A A'. In the $x-n_{1}-n_{2}-x$ tests, with $x$ $=3$ and $n_{1}=n_{2}=2$, the sequence was $\mathbf{A ~ A}^{\prime} \mathbf{A} \mathbf{A} \mathbf{A}^{\prime} \mathbf{A}^{\prime} \mathbf{A}$ $A^{\prime}$. The main result was that thresholds were substantially lower with the $x-n_{1}-x$ paradigm than the complementary $x-n_{1}-n_{2}-x$ paradigm, and that the flanking signals were relatively nondifferential in interference.

\section{Spatial Factors}

Might we overcome complementary field masking by spatial displacement of the second field relative to the 
first? What is the interaction of spatial and temporal displacement fields? Experience with photographic positives and negatives suggests that sharp outlines may emerge when complementary fields are slightly displaced.

Figure 9 presents 1-D constraint thresholds for displays with an interdot spacing of $1.17 \mathrm{~mm}$. The left panel of Fig. 9 considers tests in which one 16 by 16 field was followed by wait, $W$, before presentation of its complement. The wait interval is scaled on the abscissa; the $x$ and $y$ spacing, $s$, is the parameter and is expressed as a proportion of the intercolumn and interrow spacing of $1.17 \mathrm{~mm}$. Thus, $\mathrm{s}=0.2$ signifies a shift of $0.22 \mathrm{~mm}$. At a seating distance of $75 \mathrm{~cm}$, this is equivalent to a visual angle of $1 \mathrm{~min}$. Performance improves as the complementary pairs are pulled apart either in space or in time. With a sufficiently large wait interval, $W=$ $16 \mathrm{msec}$, spatial separation becomes relatively unimportant; with a sufficiently large spacing, $s=0.8$ or $0.94 \mathrm{~mm}$, temporal spacing becomes relatively unimportant. The middle panel of Fig. 9 shows that successive repetitions of the cycle of a 16 by 16 display field and its complement do not provide large gains in sensitivity for the 16 by 16 complementary pair, although there is a small gain with successive repetitions at narrow spatial displacements. The right panel shows the improvement in performance with longer display durations paired with spatial displacement.

Figure 10 considers thresholds for 2-D constraints with spatial separation. The organization of Fig. 10 parallels that of Fig. 9. The $2-\mathrm{D}$ constraints are more sensitive to the spatial, as well as to the temporal, separation. Otherwise, the points noted for 1-D constraints are also achieved for 2-D constraints.

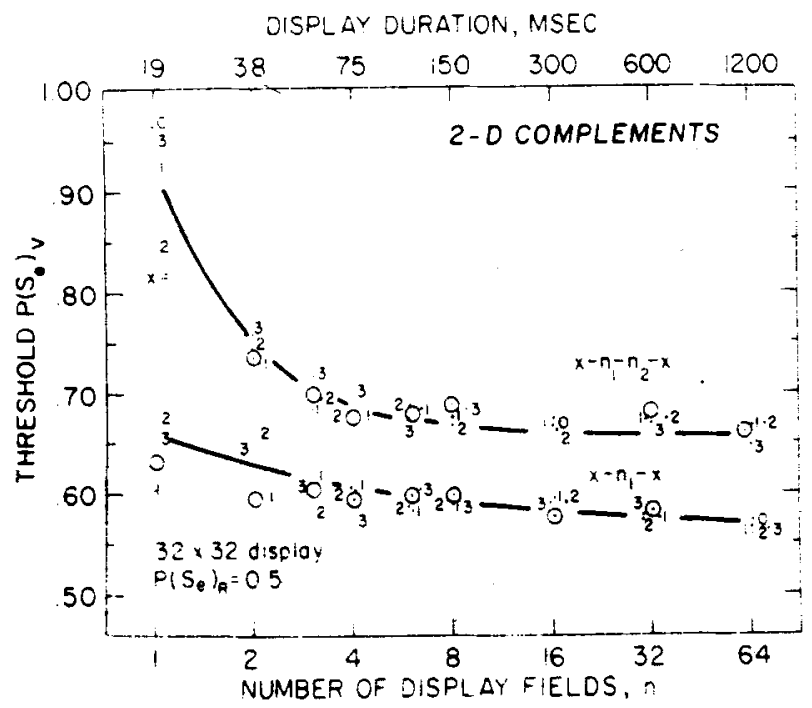

Fig. 8. Effect of flanking interference on the discrimination of two-dimensional constraints within two experimental paradigms. The parameter represents flanking interference in terms of forward (1), backward (2), forward and backward (3). or no (0) flanking interference.

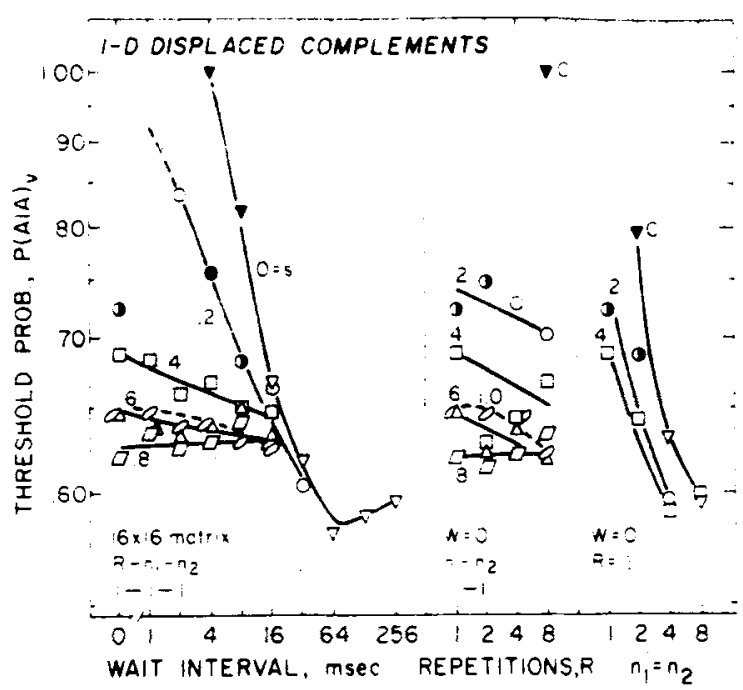

Fig. 9. Effect of spatial displacement on the detection of one-dimensional constraints within complementary pairs. The parameter is the intercolumn and interrow spacing of $1.17 \mathrm{~mm}$. The left section shows the interaction of temporal displacement (abscissa) and spatial displacement; the middle section shows the effect of successive repainting of each complement.

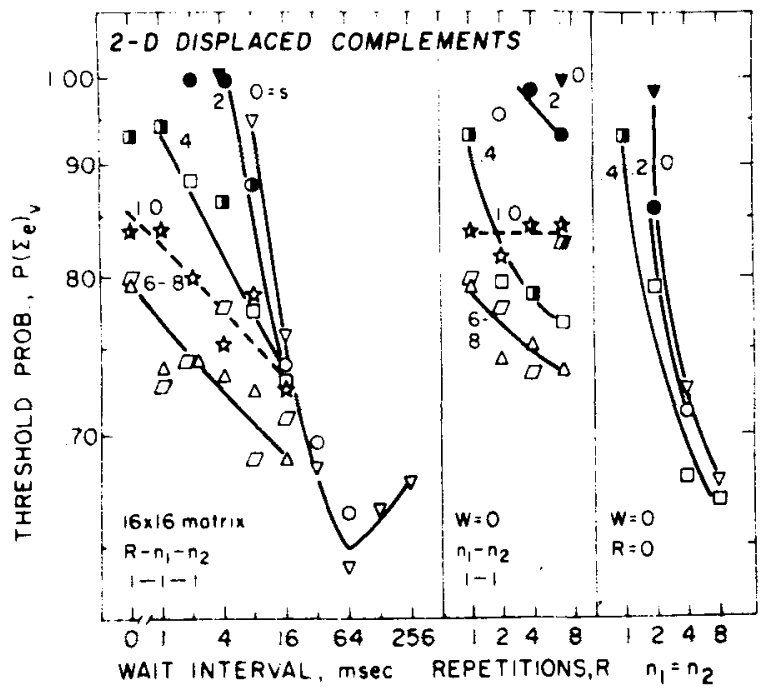

Fig. 10. Effect of spatial displacement on the detection of two-dimensional constraints within complementary pairs. Organization is as in Fig. 9.

\section{DISCUSSION}

Following Sperling (1967), one information-processing approach to visual interference holds that the presentation of a postexposure field "stops" the processing of a display field. This otherwise attractive approach suffers in application to complementary-field interference because. had the processing of the first display field been stopped. the complement field alone would have been sufficient for performing the task. Nor ean we relate visual 
interference in the complementary-field task to the spatial overlap of a display and a postexposure field (Uttal, 1970). There is no overlap of display elements over successive displays in the complementary-field task, and yet strong interference obtains.

Complementary-field interference then leads us to a view very much like Eriksen's (1966) concept of luminous summation. A postexposure field will or will not interfere with the perception of a display, depending upon the temporal-spatial properties of the display and depending upon the operator's task. In the Eriksen-Collins paradigm, where successive items must be combined to perform the task, luminance summation would aid performance at short IFIs. In the complementary fields paradigm and in the masking paradigm, it is crucial to separate the two fields to perform the task, and the lack of luminance summation at large IFIs is critical. In support of this classification, the critical temporal region for complementary-field interference approaches that for the Eriksen-Collins paradigm for a related task. ${ }^{1}$

Finally, we note that threshold performance is determined largely by the interdisplay interval-the sum of the display duration plus the interfield interval. This result suggests that equivalent processing takes place during presentation of the display and in the interfield interval. As such, the critical variable is akin to Massaro's (1972) "perceptual processing time" in audition.

\section{REFERENCES}

Eriksen. C. W. Temporal luminance summation effects in forward and backward masking. Perception \& Psychophysics, 1966. 1, 87-92.

Eriksen, C. W.. \& Collins, J. F. Some temporal characteristics of visual pattem perception. Journal of Experimental Psychology. 1967. 74, 476-484.

Massaro. D. Perceptual images. processing time, and perceptual units in auditory perception. Psychological Review. 1972, 79. 124-145.

Mayzner, M. S.. \& Greenberg, J. Studies in the processing of sequentially presented inputs with overprinting paradigms. Psychonomic Monograph Supplements. 1971. 414. Whole No. 52), 73-84.

Pollack, I. Visual discrimination thresholds for one- and two-dimensional Markov spatial constraints. Perception \& Psychophysics, 1972, 12, 161-167.

Sperling, G. A model for visual memory tasks. Human Factors, $1963,5,19-31$.

Uttal, W. R. On the physiological basis of masking. Perception \& Psychophysics, 1970, 7, 321-327.

\section{NOTE}

1. Pollack, I. Interaction effects in successive visual displays: An extension of the Eriksen-Collins paradigm. Submitted for publication.

(Received for publication August 2, 1972; accepted for publication November 1, 1972.) 